\title{
Laparoscopic Resection of Metastatic Pelvic Liposarcoma
}

\author{
Hong B. Shim, Tae Y. Jung, Ja H. Ku \\ Department of Urology, Seoul Veterans Hospital, Seoul, Korea
}

\begin{abstract}
We report a pelvic liposarcoma originating from the left spermatic cord that recurred following inadequate excision. In our case, the tumor was resected without performing orchiectomy previously. The patient was managed by laparoscopic resection, before undergoing radical orchiectomy in the left inguinal region. To our knowledge, no case of laparoscopic resection for the recurrent liposarcoma has been described. In addition, the present case serves to demonstrate that radical orchiectomy with wide excision is needed for paratesticular tumor.
\end{abstract}

Key words: spermatic cord; liposarcoma; metastasis; pelvis; laparoscopy

Int Braz J Urol. 2006; 32: 445-7

\section{INTRODUCTION}

Paratesticular liposarcomas are rare malignant mesenchymal tumors that develop in fatty tissues. The choice of therapy for paratesticular liposarcoma is surgical, through orchiectomy and extensive local removal. We report here a pelvic liposarcoma originating from the recurrent paratesticular liposarcoma. We emphasize that the tumor should be treated with radical orchiectomy with high ligation of the cord and the patients must be followed periodically on a life-long basis.

\section{CASE REPORT}

A 73-year-old man presented at our hospital with a palpable mass at the left inguinal area. The patient had been treated for a paratesticular mass with resection via a high inguinal approach 7 years previously at other hospital and had been told that histological diagnosis at that time had been liposarcoma.
Physical examination revealed a firm non-tender immobile mass at the left inguinal area, which was the same region of the primary mass and an upward displacement of left testis. Hematologic examination and urinalysis were not remarkable.

He underwent computed tomography of the abdomen and pelvis, which demonstrated a capsulated fat-like heterogenous mass measuring $3.3 \times 3.3$ x $2.0 \mathrm{~cm}$ adjacent to sigmoid mesocolon and a partially fat one containing soft tissue mass measuring $4.1 \times 3.5 \times 3.0 \mathrm{~cm}$ in the left inguinal area (Figure-1). No other metastatic lesions were evident on abdominal and pelvic CT. The pelvic mass was removed laparoscopically, while the inguinal mass was resected en bloc during inguinal radical orchiectomy. The patient was placed in a supine position. After creating space with carbon dioxide insufflation, three ports were placed. The pelvic mass was an isolated nodule and thus it was not necessary to ligate any major vessel to avoid opening the mesocolon for complete resection of the mass. The specimen was trapped in a sterile plastic bag and removed intact via a port site 

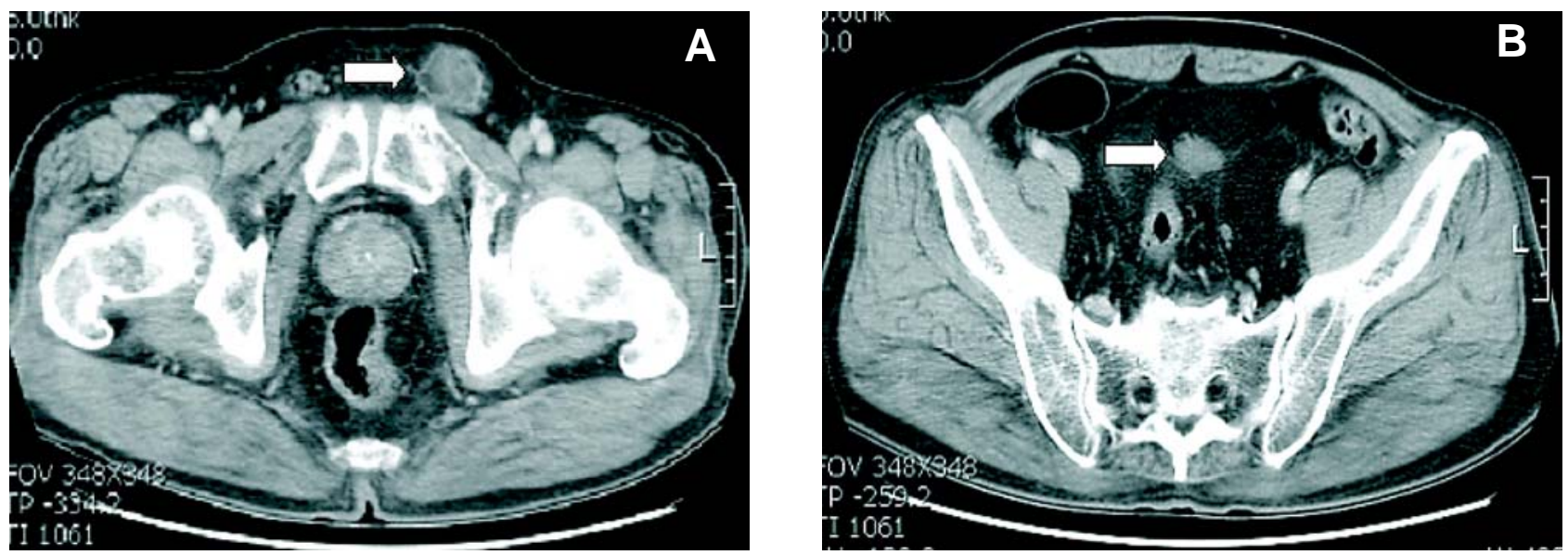

Figure 1 - Contrast-enhanced computed tomography of the pelvis. A) Partially fat containing soft tissue mass (arrow) at the left inguinal area. B) Homogenous enhanced soft tissue mass (arrow) adjacent to sigmoid mesocolon.
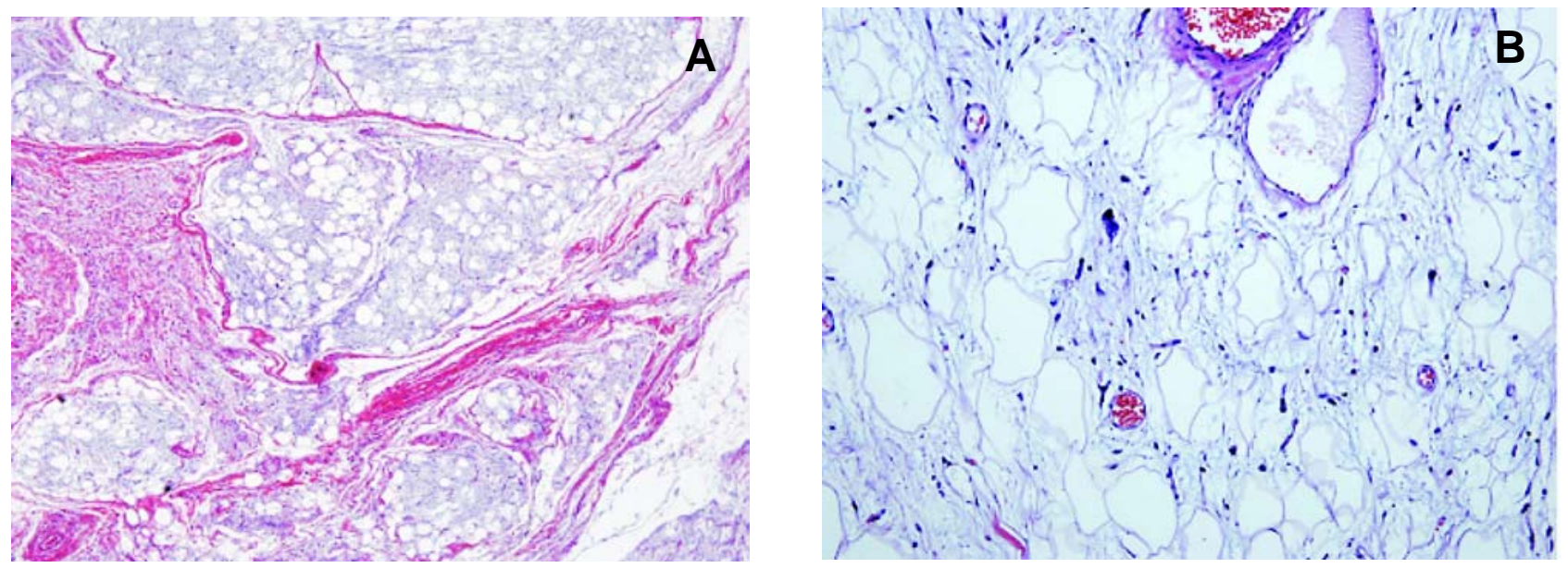

Figure 2 - Pathological findings of the tumor are indicative of myxoid liposarcoma. A) Paratesticular liposarcoma (HE, X40). B) Pelvic liposarcoma (HE, X200).

after an additional $1 \mathrm{~cm}$ incision. Pathological examination of resected specimens in both pelvis and left inguinal area revealed myxoid liposarcoma (Figure2). Surgical margins were free of tumor. Without any postoperative adjuvant therapy, no evidence of recurrence or metastasis was noted during the 6-month follow-up period.

\section{COMMENTS}

Liposarcoma is histologically subdivided into 3 types, including well differentiated, myxoid and pleomorphic. Myxoid liposarcoma tends to occur in middle age, generally between 40 and 60 years of age. It tends to recur locally, due to its intermediate malignant behavior but it rarely metastasizes to distant organ. Since myxoid liposarcoma is the most radiosensitive type of all liposarcomas, adjuvant radiotherapy has been recommended if local control is not complete. Radiotherapy is also recommended in addition to surgery in cases evidencing a more aggressive tumor behavior (i.e., high-grade tumor, lymphatic invasion, inadequate margin or recurrence). Retroperitoneal lymphadenectomy does not offer any addi- 
tional therapeutic benefit, and the role of chemotherapy is not well defined.

A case of port site metastasis after laparoscopy-assisted surgery for retroperitoneal liposarcoma has been previously reported. A possible mechanism for port site cell implantation is a length operative procedure with high-pressure penumoperitoneum, which enables the tumor cell to implant locally or disseminate hematogeneously during the operation (1). Our case is the first description of pelvic liposarcoma metastasizing from the inguinal area, which was managed by laparoscopic procedure. In this case, there was no difficulty or special care during the laparoscopic procedure.

A case of liposarcoma has been described which was treated successfully by tumor excision without involvement of the testis (2). However, simple excision may be inadequate treatment for paratesticular sarcoma, since wide repeat excision may reveal microscopic residual disease in some of completely excised cases (3). Our finding emphasizes that in the treatment of spermatic cord liposarcoma complete mass resection and inguinal orchiectomy with high ligation of the spermatic cord must be per- formed. However, as the histopathology of the earlier surgery was not available, the possibility of malignant change in a preexisting lipoma could not be excluded. Regardless of initial therapy, the risk of recurrence of paratesticular liposarcoma always necessitates long-term follow-up.

\section{CONFLICT OF INTEREST}

None declared.

\section{REFERENCES}

1. Martinez J, Targarona EM, Balague C, Pera M, Trias M: Port site metastasis. An unresolved problem in laparoscopic surgery. A review. Int Surg. 1995; 80: 315 21.

2. Crespo Atin V, Padilla Nieva J, Martin Bazaco J, Llarena Ibarguren R, Pertusa Pena C: Scrotal liposarcoma. Arch Esp Urol. 2001; 54: 729-32.

3. Catton C, Jewett M, O'Sullivan B, Kandel R: Paratesticular sarcoma: failure patterns after definitive local therapy. J Urol. 1999; 161: 1844-7.

Accepted after revision:

March 6, 2006

\author{
Correspondence address: \\ Dr. Ja Hyeon $\mathrm{Ku}$ \\ Dept Urology, Seoul Veterans Hosp \\ 6-2, Doonchon Dong, Kangdong-Gu \\ Seoul 134-791, Korea \\ Fax: + 8224834260 \\ E-mail: randyku@hanmail.net
}

